\title{
Selection and Re-Selection in Stochastic Democracy
}

\author{
Peter J. Schubert, Ph.D., P.E. \\ IUPUI, Indianapolis, IN 46202
}

\begin{abstract}
Self-governance of space habitats using Stochastic Democracy is objective and corruptionresistant. Prior simulation studies compared Stochasticism with elitism, pure communism, and pure capitalism for long-duration ark ships, demonstrating superior outcomes for all individuals. Additional simulations explored resilience to decimation, and to rapid population growth, such as may be experienced by large space settlements. In this study, the initial population of a hierarchical governing structure is simulated, providing a procedure for start-up on Day 1. Furthermore, rules for re-selection are explored to provide a balance of fresh inputs and experienced leadership. A key tenet of Stochastic Democracy is preclusion of career politicians who can be unduly influenced by campaign contributions and unduly favored by redistricting ("gerrymandering"). However, preserving learning within a governmental hierarchy is valuable, so the re-selection process should include a means by which selected leaders either retain office or advance to greater levels of geographic and population responsibility. Staggering of terms is another important means by which some level of continuity of governance is retained. In this work, several alternatives are explored to provide a sense of the impacts of re-selection rules. It is conceivable that individual communities may vote on referenda which capture their preferences for re-selection rules and duration of terms. In this way, Stochastic Democracy becomes adaptable to the community sociology and to possible future changes in lifespan. This work completes earlier AIAA-published theoretical studies of this novel form of habitat self-governance.
\end{abstract}

\section{Introduction}

$\mathrm{T}$ he SPACE 2010 and SPACE 2013 publications upon which this work is based ${ }^{1,2}$ introduced and explored a model of a corruption-resistant form of representative government based on stochastic selection within preestablished constraints. The first study compared Stochastic Democracy with three forms of governance: elitism, where $15 \%$ of the population control a disproportionate share of overall wealth; capitalism, a merit-based system where a minimal government provides infrastructure and order; and pure communism where benefits are distributed regardless of individual effort or ambition. Each of the 4 governance systems was applied to an agent-based simulation (populace and government) of a closed habitat isolated for 10 years (e.g. an ark spaceship). Stochastic Democracy was found to be superior in lives preserved, smallest number of impoverished citizens, and reasonable distribution of wealth. Capitalism was found to perform nearly as well with lives preserved but evolved a bimodal wealth distribution similar to that of elitism. Communism fared poorly on all figures of merit. The second study explored hierarchical districting using the method of k-means. The resiliency of this approach was tested by subjecting the population to either massive immigration or decimation. Changes in population density over 2 orders of magnitude were readily adapted to by the 2-level government hierarchy for a population of 50,000. Those results also demonstrate the ability of Stochastic Democracy to scale up from a minimal population of about 4,000 to a population of any size, even spanning multiple planets and, eventually, multiple star systems. What has been missing up to this point is an exploration of how Stochastic Democracy gets started. Specifically, how does one create a complete government hierarchy on Day 1 when office holders all start at the same time and then transition smoothly to a steady state with staggered terms of service?

In this work a population of 3,500,000 living within a square area of 37.5 x 37.5 kilometers adopts Stochastic Democracy on Day 1 for governance hierarchy having 4 levels, as determined by the formula in reference 2 . The top level (4) has 9 leaders, and lower levels have powers of 9, namely: 81 for level 3; 729 for level 2; and 6,561 for level 1. Law-makers are selected from a pool of volunteers, assuming that $4 \%$ of the population both volunteers and meets the minimum requirements for service, namely: (1) no felony record; (2) never declared personal bankruptcy; and (3) completed a 4 year program such as a college degree or equivalent military service. These requirements are not meant to be elitist, but rather are screening tools to preclude known criminals, poor fiscal managers, and those for whom completing a multi-year program (such as government leadership) has not been demonstrated. Terms of service are 3 years at each level, however in Years 1, 2, and 3, some terms are abbreviated to achieve staggering.

1

American Institute of Aeronautics and Astronautics

This is the author's manuscript of the article published in final edited form as:

Schubert, P. J. (2015). Selection and Re-selection in Stochastic Democracy. In AIAA SPACE 2015 Conference and Exposition (p. 4606).

http://dx.doi.org/10.2514/6.2015-4606 


\section{Method}

Continuity within the leaders of government provides for long-term planning and allows established representatives to train newly-selected ones during term overlap. Those governments where undue influence exists, and where re-districting is subject to political fortunes (gerrymandering) generally have strong continuity. Where re-election is a very high probability (the U.S. Congress being a very good example) there is incentive for wealthy individuals, cabals, or corporations to establish close relations with leaders often in anticipation of favorable consideration. While such systems preserve the status quo effectively, the degree to which they fairly represent the entire constituency is limited. At the opposite extreme, rapid changeovers of entire governments is inefficient and raises concerns of uncertainty which can dampen enthusiasm for investments in business and infrastructure. Stochastic Democracy aims to balance these factors through two inter-woven methods: stochastic selection, and reselection rules, which are the primary focus of the current work.

\section{A. Stochastic Selection}

A stochastic process is one in which the evolution over time of some system of random variables is unpredictable and there are an almost infinite number of potential outcomes. Nevertheless, it is possible to characterize responses and derive response bounds approached as a limit. Starting Stochastic Democracy on Day 1 is the Initial Selection process in which pre-qualified volunteers are grouped by district for the various levels of a government hierarchy and then selected at random from the pool of local candidates until the leadership positions are all filled. This is the only time selection is so easy, at Day 1 of Year 0 . Figure 1 shows the spatial distribution as color-coded markers on a square grid with 10 meter increments. Level 1 is shown as red dots, level 2 as blue diamonds, level 3 as black triangles, and level 4 as large green star-shapes.

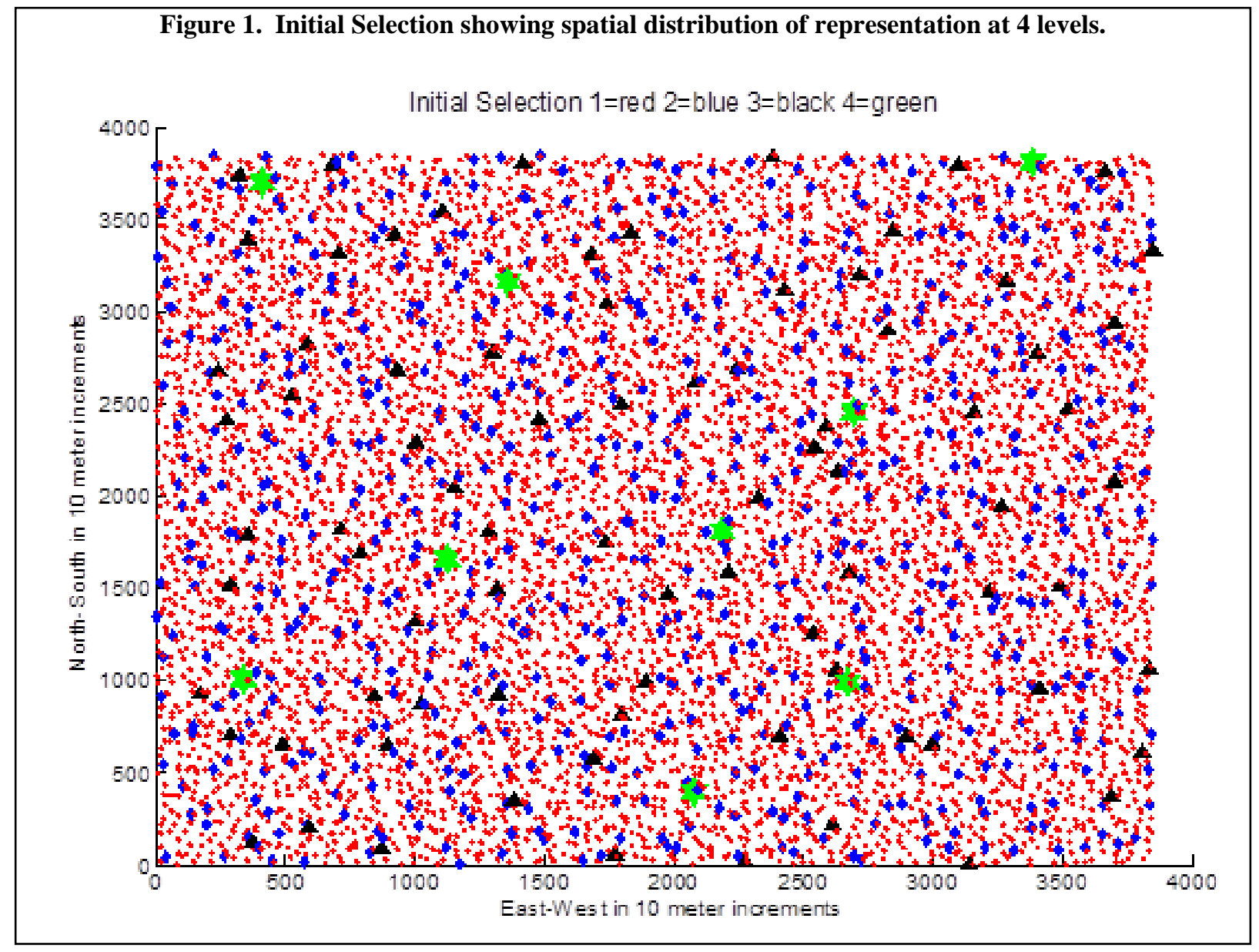


A simple method for transition from an all-at-once start to an on-going steady state in which $1 / \mathrm{N}$ positions are exchanged each year within an $\mathrm{N}$ year service term is to abbreviate a 1/N portion of the Day 1 appointments at Year 1 , and another 1/N portion at Year 2, and so on to Year N. For this work, the transition years up to Year $\mathrm{N}$ are individually coded and unique. Steady state begins at year $\mathrm{N}+1$, and a common algorithm is used for every subsequent year. This sequence is illustrated in Figure 2.

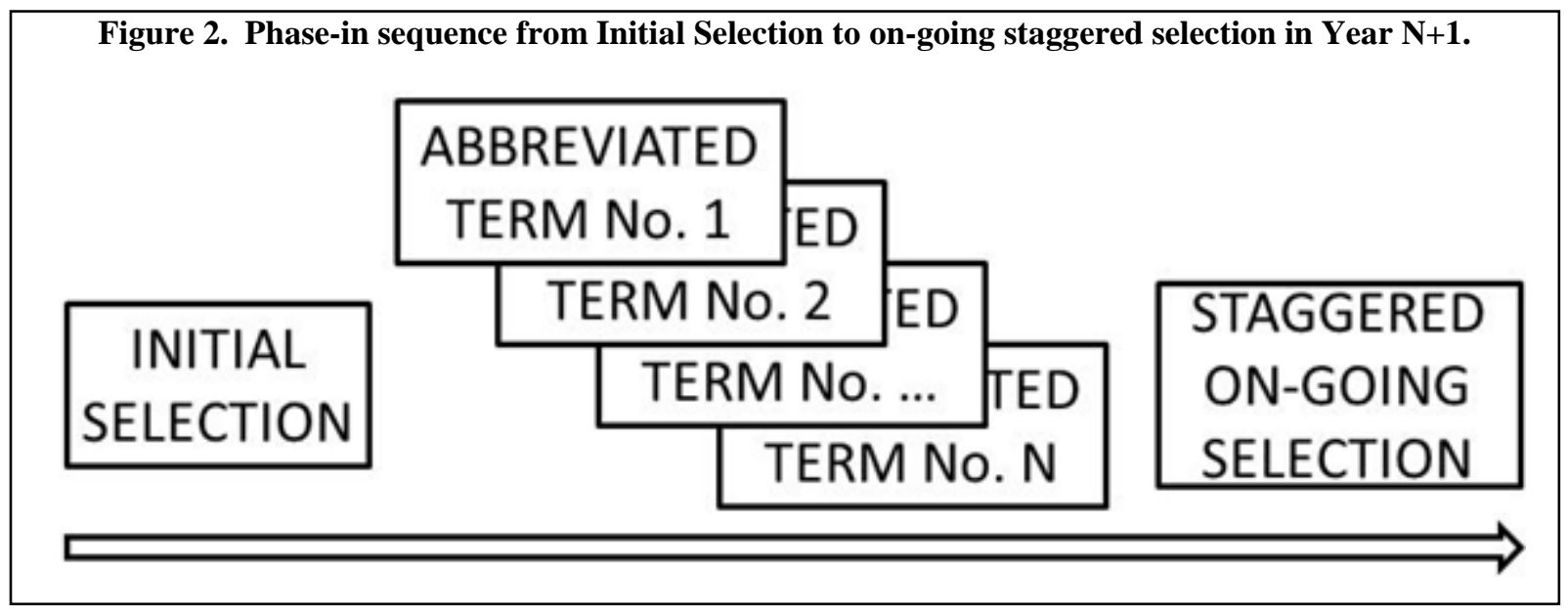

\section{B. Re-Selection Rules}

Preservation of continuity of government is accomplished in Stochastic Democracy by comparing a random variable within probability bins to determine the treatment of an individual decision-maker upon completion of a term of service. There are five possible outcomes:

1. Re-selected for service at the same level (STAY),

2. Return to the local candidate pool but still remain eligible for later selection (OUT),

3. Retire from service, returning to the general population (RETIRE),

4. Be considered for a subsequent term at a level lower within the hierarchy (DOWN), and

5. Be considered for a subsequent term at a higher level within the hierarchy (UP).

Figure 3 shows a state machine for Re-selection. The lowest level has the STAY, OUT, and UP options; the top level has STAY, OUT, and DOWN options; and all intermediate levels have STAY, OUT, DOWN, and UP options. The RETIRE option is not shown but is applied programmatically in the simulation. Note the presence of two temporary candidate pools, the UP POOL and the TRANSITION POOL. These are temporary asignations at the end of Re-selection and accessed again during the Vacancy Fill operation immediately after.

Figure 4 shows the probabilities used for the baseline Reselection Process used from Year $\mathrm{N}+1$ onward. The allocation of probability bins in Fig. 4 are arbitrary. In subsequent sections these baseline probability bins are modified to explore their impact on continuity of government and representation of the citizenry.

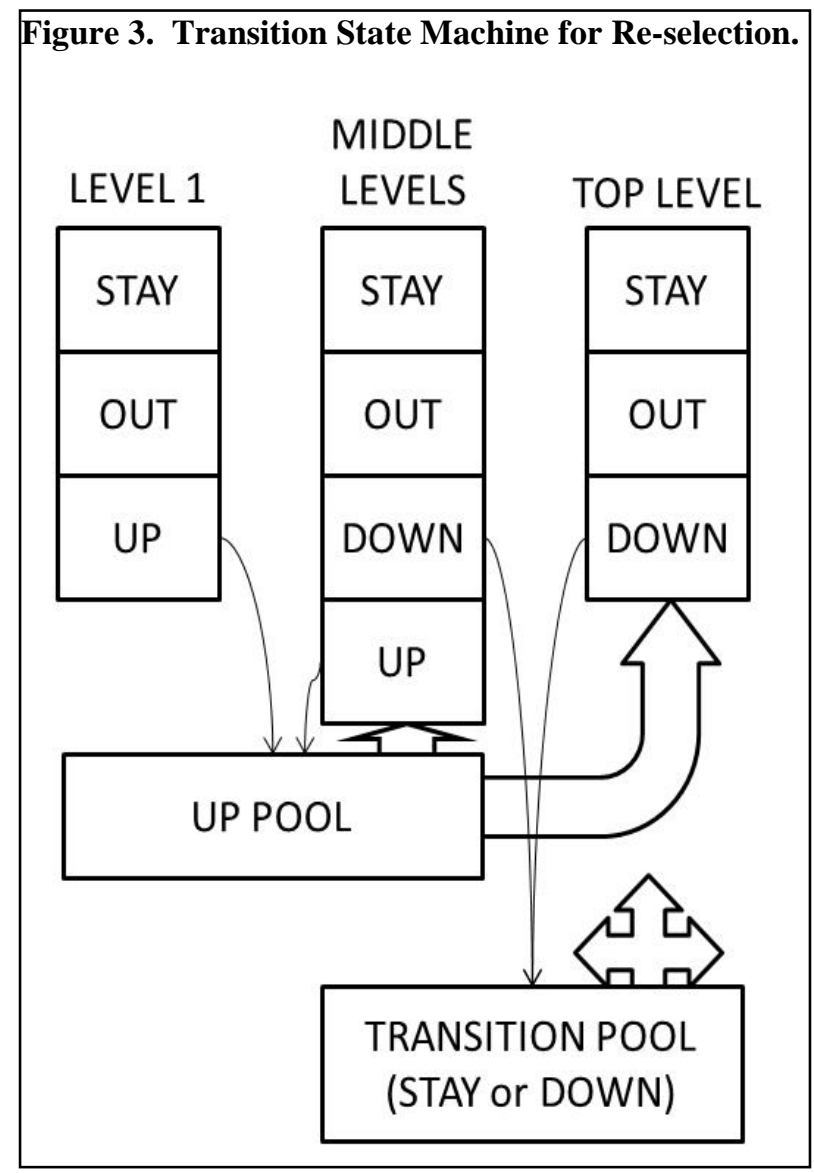




\section{Vacancy Fill}

When Re-selection results in STAY, OUT, or RETIRE, the action is immediate. Those moved OUT of office remain active volunteers in the local candidate pool, and as will be shown below, are sometimes selected again after many years. Of course volunteers can remove themselves (RETIRE) at any time.

The number of vacancies at each level of the hierarchy is thus $1 / \mathrm{N}$ of the total offices minus those who are reselected to STAY. Filling the remaining vacancies is then distributed among (as appropriate) the UP POOL, the TRANSITION POOL or the local candidate pool according to a heuristic described in flow chart form in Figure 5.

The Vacancy Fill operation, immediately following the Re-selection process starts at the top level to fill those vacancies not filled by a STAY selection. The next priority is those from the UP POOL having just completed a term of service at the next level lower. This provides a means by which those experienced at a given level of government may bring their experience to a wider constituency.

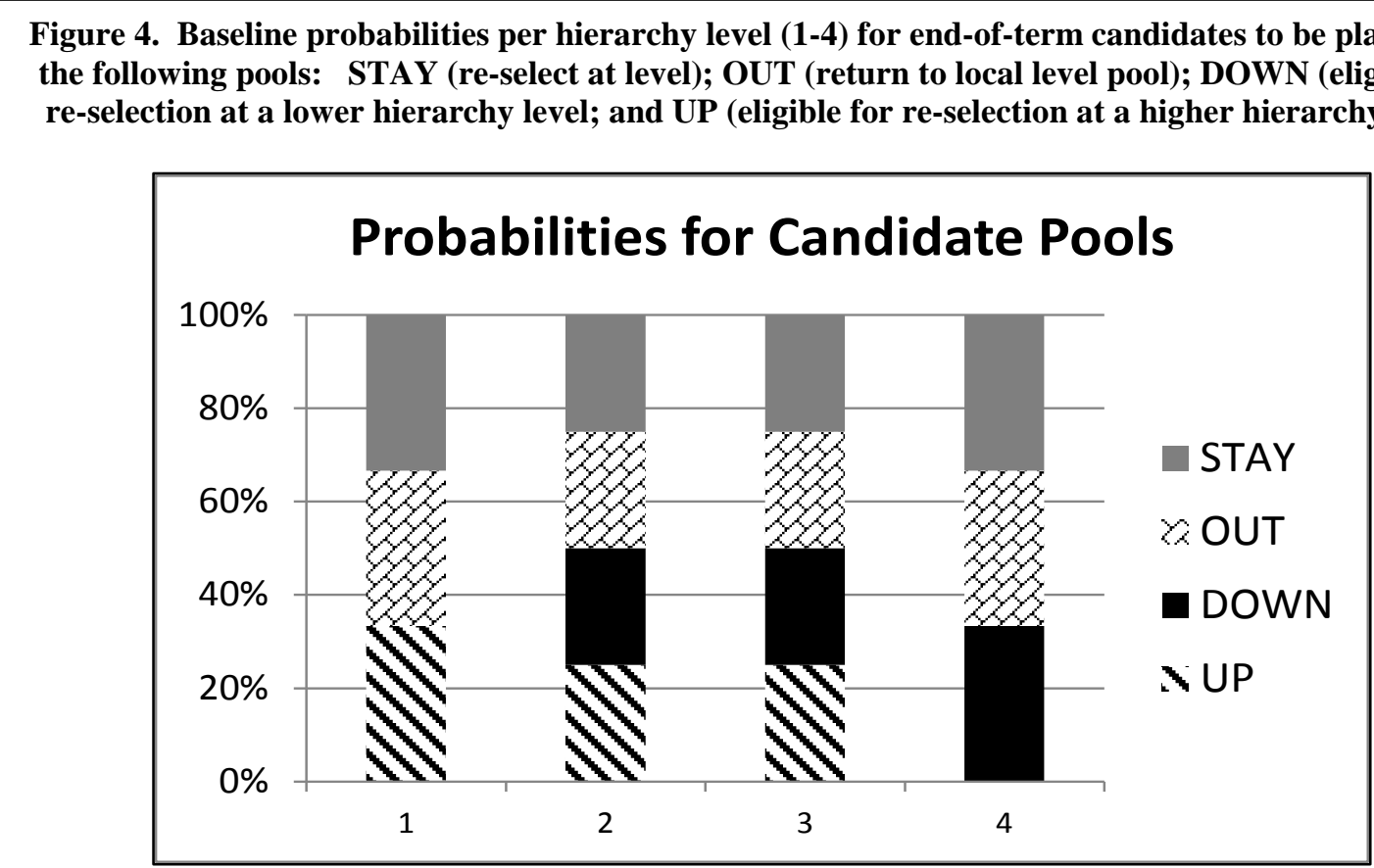

The subsequent priority is to fill any remaining vacancies (unlikely) from the UP POOL from the level 2 below the top level, et. seq. until any remaining needed vacancies are filled from the local candidate pool. For intermediate levels, the Vacancy Fill process begins immediately after the Re-selection and assigns first priority to UP POOL candidates from one level lower, provided they are in the proper district. Second priority is assigned to those in the DOWN POOL from one level higher, again assuring residency in the instant district. This latter process is a means by which more broadly-experienced representatives may bring their perspective and expertise to a more focused constituency, perhaps aiding in harmonizing the entire hierarchy of government. 


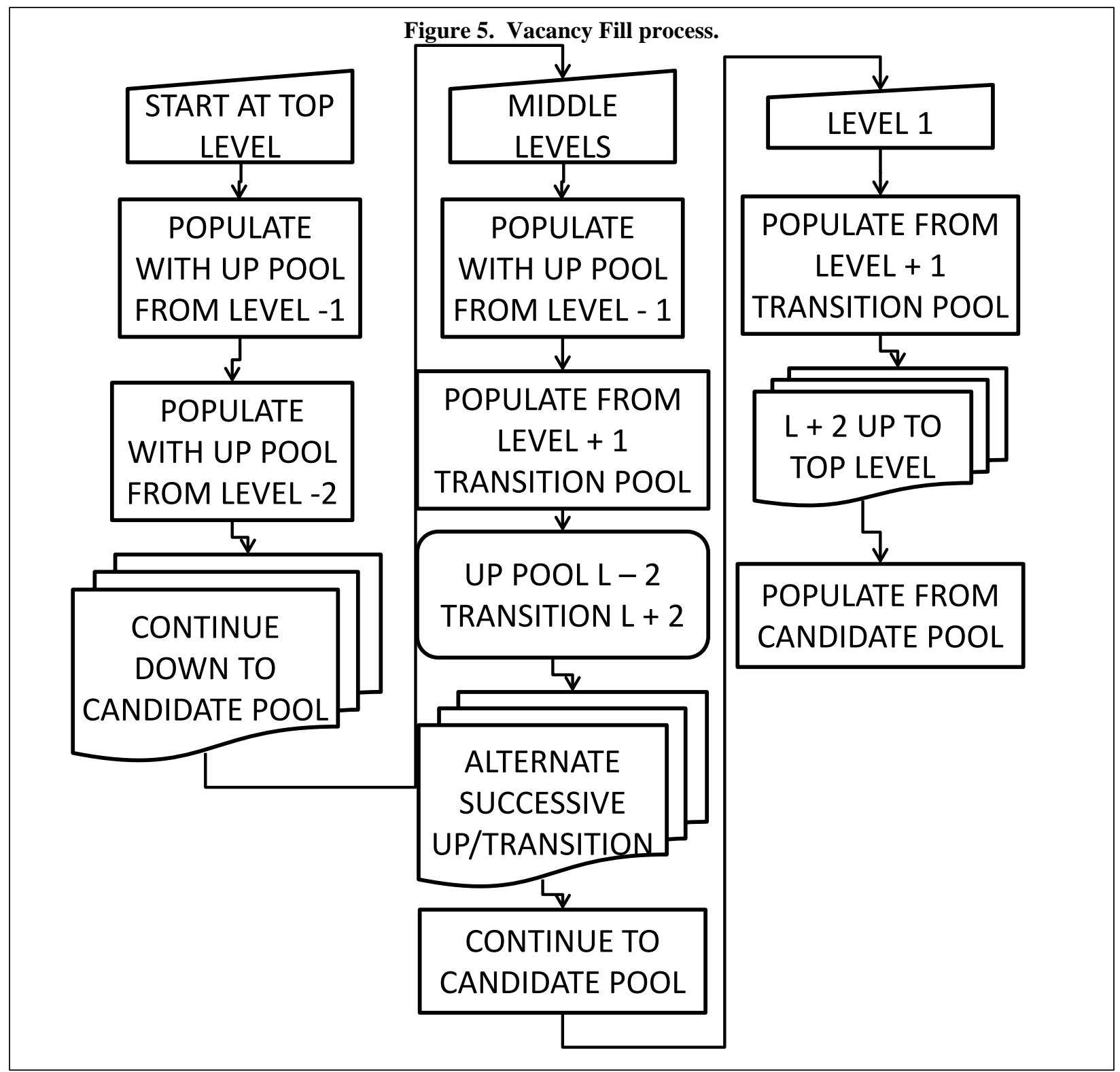

Further remaining vacancies at the intermediate levels alternate between successively lower levels and successively higher levels, always with the proviso that the candidate be within district, until the highest level has been mined and the local candidate pool has been mined as well. At the lowest, most local level of government candidates are drawn from level 2, if in district, and if further vacancies remain, from level 3 et. seq. to the top level. Finally, if there still remain vacancies at level 1 they are drawn from the local candidate pool. Being the most local level, and thus restrictive of candidates from higher levels (predominantly from other local districts), it is relatively more likely that fresh candidates will thusly enter service at level 1.

\section{Results}

All simulations were performed with MATLAB R2011B ${ }^{3}$ using M-scripts. All service terms were set at three (3) years regardless of level. After Initial Selection and the transition years up to Year 4, subsequent Year $\mathrm{N}$ simulations were approximately 150 minutes in duration on a standard desktop computer. A total of 21 years were simulated. Figure 6 shows representative validation results showing the number of the prior level for level 3 
selectees at Year 1 (one year from Day 1). As expected from Fig. 4, very nearly 1/3 of the level 3 appointees from Day 1 were re-selected to serve a term to run the full 3 years. The remainder all come from level 2 (UP POOL). For these 81 leadership positions, no candidates were required from level 4 , level 1, or the local candidate pool.

From Year 1 to Year 21 the number of vacancies for level 4 positions were tracked. With 9 position to fill and $1 / 3$ of the representatives' terms expiring each year there will be 3 vacancies. From Fig. 4 there is a 1 -in-3 probability for an expiree being re-selected for level 4 , so the expected value for vacancies is 2 . Figure 7 shows the actual result for the years studied. The average number of vacancies each year for level 4 was 1.3. Note that zero vacancies is a 1 -in- $3^{3}$ event and should be expected once

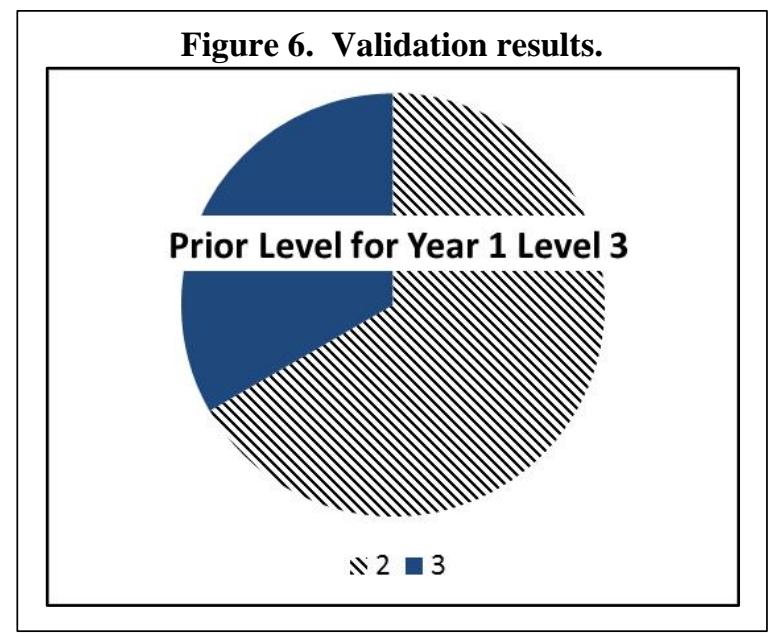
(maybe twice) in a 21 year simulation.

Table 1 shows the end-of-year vacancy numbers for each level averaged across 21 years, including the standard deviation value. Note that the standard deviation value drops asymptotically to the large $\mathrm{N}$ limit as would be expected of a stochastic process.

Table 2 shows selected individuals having been stochastically assigned interesting or illustrative service records. The first example called "Founder Returns" shows an

Figure 7. Distribution of end-of-year (EOY) vacancies for Level 4 after re-selection. Total leadership at level 4 is 9 members.

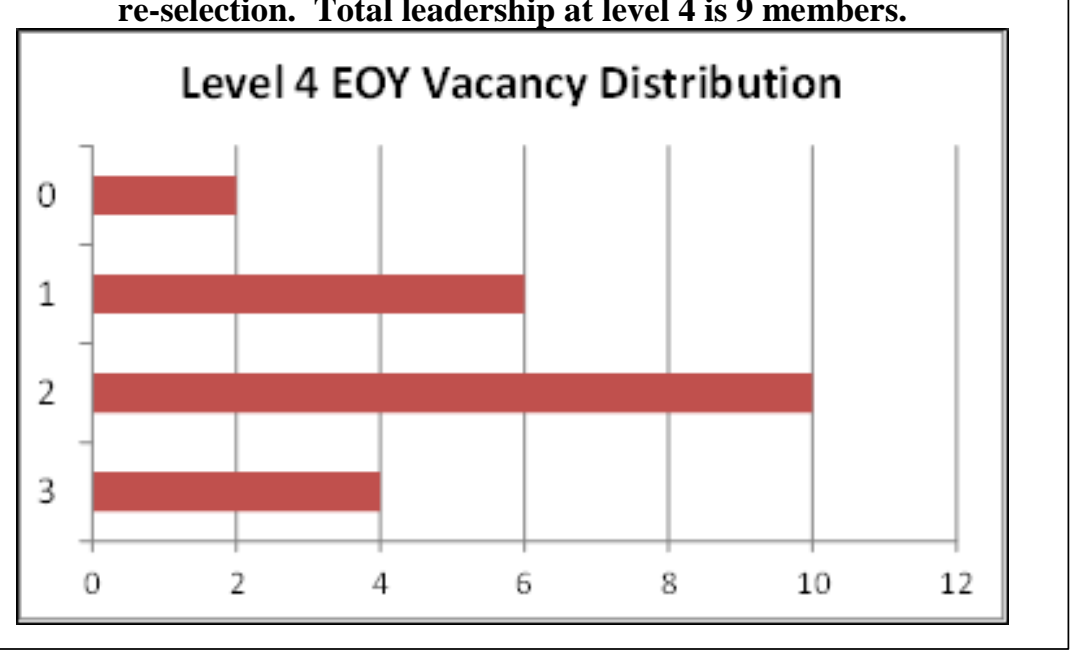

individual serving only 1 year during the start-up of the government. This was followed by another 1 year of no service, but still assigned within the local candidate pool. In the next year, this individual was again selected to level 1 and served a normal 3 year term of service. From then until the end of the simulation run this individual remained in the local candidate pool and was not selected for further service. The bottom right "Service Career" individual has 20 years of service and most closely approximates the "career politician" of a government with high degree of continuity. Of a population consisting of 3.5 million, this is a rare, but not impossible, scenario.

Table 1. End of year re-selection average and standard deviation over 21 years. Column 4 shows total vacancies per year. Vacancies not filled by re-selection are drawn from various candidate pools as described elsewhere herein.

\begin{tabular}{|c|r|r|r|}
\hline LEVEL & AVERAGE & ST.DEV. & VACANCIES \\
\hline 1 & 789 & 318 & 2187 \\
\hline 2 & 70 & 39 & 243 \\
\hline 3 & 7.0 & 5.2 & 27 \\
\hline 4 & 1.3 & 0.88 & 3 \\
\hline \hline
\end{tabular}


Table 2. Selected individuals (out of 3.5 million) with illustrative or interesting service records.

\begin{tabular}{|l|c|c|c|}
\hline "Founder Returns" & LEVEL & YEARS & SINCE \\
\hline Initial Selection & 1 & 1 & 1 \\
\hline From Local Pool & 1 & 3 & 16 \\
\hline
\end{tabular}

STORY: One year during government start-up, in pool 1 year, then 1 full term, then 16 year since

\begin{tabular}{|l|c|c|c|}
\hline "Long Break" & LEVEL & YEARS & SINCE \\
\hline Initial Selection & 1 & 3 & 0 \\
\hline Re-select & 1 & 3 & 14 \\
\hline From Local Pool & 1 & 0 & \\
\hline $\begin{array}{l}\text { STORY: After } 6 \text { years as a founding leader, } 14 \text { years } \\
\text { pass before the next round of service }\end{array}$ \\
\hline
\end{tabular}

\begin{tabular}{|l|c|c|c|}
\hline "Up Then Down" & LEVEL & YEARS & SINCE \\
\hline From Local Pool & 1 & 3 & 0 \\
\hline from Up Pool & 2 & 3 & 0 \\
\hline From Up Pool & 3 & 3 & 0 \\
\hline Re-select & 3 & 3 & 0 \\
\hline Re-select & 3 & 3 & 0 \\
\hline from Down Pool & 1 & 3 & 0 \\
\hline Re-select & 1 & 3 & 0 \\
\hline $\begin{array}{l}\text { STORY: Effects a rise to higher offices, including 9 } \\
\text { years at Level 3, then 6years back at Level 1 }\end{array}$ \\
\hline
\end{tabular}

\begin{tabular}{|c|c|c|c|}
\hline "Service Career" & LEVEL & YEARS & SINCE \\
\hline Initial Selection & 3 & 1 & 0 \\
\hline From Up Pool & 4 & 3 & 0 \\
\hline Re-select & 4 & 3 & 0 \\
\hline Re-select & 4 & 3 & 0 \\
\hline Re-select & 4 & 3 & 0 \\
\hline from Down Pool & 1 & 3 & 0 \\
\hline Re-select & 1 & 3 & 0 \\
\hline \multicolumn{4}{|c|}{$\begin{array}{l}\text { STORY: } 20 \text { years of service, including } 3 \text { re-selects } \\
\text { at Level } 4 \text { ( } 3.7 \% \text { probability). }\end{array}$} \\
\hline
\end{tabular}

Across 21 years simulating the operation of Stochastic Democracy those persons who volunteered to be a candidate spent on average 2.10 years in service. With a total of 7380 governmental leadership positions, a bit over 22,000 held office at least once. Of the 147,000 volunteers (4\% of 3.5M) 125,000 were never selected using the baseline probability bins from Fig. 4, or a $89 \%$ chance of never being selected. This outcome is largely a result of the relatively high probability of a STAY result from the re-selection process, which favors continuity over distributed representation. To explore this further, a simulation variant to increase representation was performed with probability bins giving much smaller opportunities for STAY and much larger opportunities for UP.

Figure 8 shows the variant re-selection probabilities and the distribution of vacancies at Level 3. The average number of vacancies immediately after Re-selection rules were applied increased by an average of 1.4 positions compared to the baseline probability distribution functions of Fig. 4, and the number of volunteers never selected was 124,000 . These differences are not very significant, indicating that the algorithms are relatively insensitive to adjustments to probability functions.

Figure 8. Re-selection probabilities for increased-representation variation, plus end-of-year (EOY) vacancies for Level 3 after re-selection. Total leadership at level 3 is 81 members.

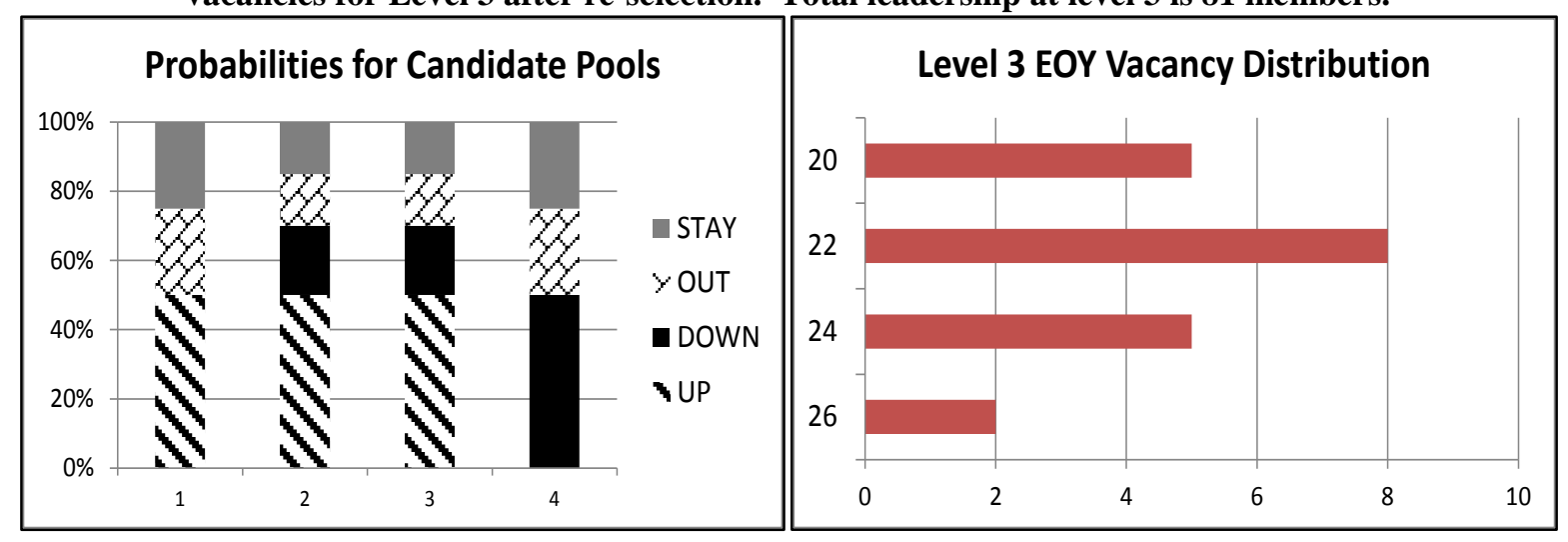




\section{Discussion}

The scenario where a fully-staffed government is started in a single day is perhaps uncommon in human history, but could be envisioned to apply to space settlement where an ark ship arrives at a recently-prepared habitat. Drawing upon the results of the prior two studies ${ }^{1,2}$, the first order of business would be for people to select their domiciles so that a census could be taken which includes the location of each residence. The k-means method of districting applies to multiple dimensions and can thus be extended to a 3D living space such as the interior of an asteroid.

Presuming that most members of such a society are engaged in some productive activity, career, business leadership, trade, or other activity where continuity of said activity has value to the enterprise being served, one must address the issue of compensation. While in practical application, a populace may form a consensus (perhaps by voting) on a compensation scheme for its leaders, one possible scheme is presented here for consideration. This proposal assumes that each individual has a history of gross income over a 3 year period prior to the start of a Stochastic Democracy start-up. Note that zero salaries are also anticipated, as may be the case for full-time students recently graduated from college, or stay-at-home parents, or indigent elderly. This proposal also assumes that the society or societies from which the population was drawn can establish a metric defining a "poverty level" or similar metric of minimal self-sufficient subsistence for self or self plus family.

The heuristic for compensation considers gross income over the prior 3 years (exclusive of suspended animation or other enforced income-generating hiatus), which may be steady, growing, shrinking, variable, or absent. Cases are listed here based on gross income over 3 years (GI3), for compensation for office-holders in-service:

1. GI3 within $+/-10 \%$ : compensation at $105 \%$ of average

a. If re-selected, regardless of level, each subsequent term at $105 \%$ of prior term's compensation.

2. GI3 zero or below poverty level: compensation at $105 \%$ of poverty level

3. GI3 highly variable: compensation at $105 \%$ of median gross income

4. GI3 declining or rising steadily more than $+/-10 \%$ : compensation at $105 \%$ of median value

If an individual is re-selected, regardless of level, each subsequent term at $105 \%$ of prior term's compensation.

Another aspect to consider when initiating a new form of governance is the desire for people to vote. In Stochastic Democracy the popular vote is not used for selection of decision-makers in the legislative body. They may be asked to vote on the compensation scheme, and this could be periodically revisited as the community's fortunes change. Terms of service may also be opened for popular vote, as for example if future medical advances permit significantly longer human lifetimes calling for longer terms of governmental service. Re-selection probabilities are also a consideration for popular vote. Even the relatively large changes in probability distributions had minimal effect on the re-selection results, so further adjustments may be an experiment left to the voice of the people. The algorithm behind the Re-selection rules is envisioned to remain fixed, as modifications may be subject to manipulation of individuals or small groups to unfair advantage.

The head of state for the government may be a position which is put to the popular vote. Should the populace wish for a single leader to represent them to other communities, or to preside over official ceremonies, or to petition the council at the top level of the government hierarchy, there may be a President elected by popular vote. Each level may have a similar figurehead for each district. Other positions which may be considered for popular vote include judges, law enforcement leaders, school boards, commissioners/engineers, and other public offices which may benefit from having popular support.

\section{Conclusion}

The results indicate that start-up of a fully-staffed Stochastic Democracy and a smooth transition to staggered terms is eminently feasible. Re-selection rules were found to be relatively insensitive to modest adjustments of probability distributions between various outcomes (STAY, UP, OUT, DOWN) following successful conclusion of a term of service. Building upon the results of the previous two studies of Stochastic Democracy, this work completes the assessment of the following characteristics:

A. Superior quality of life compared to existing forms of governance

B. Scalable to any size of community (above 4000), and ability to scale up or down rapidly 
C. Ability to start instantly and transition smoothly to steady state

D. Fairly represents all members of a community

E. Resistant to systemic corruption and undue influence of small minorities

F. Adaptable to the will of the people through parametric adjustments available to poplar vote.

A key theme behind the genesis of Stochastic Democracy is that people tend to be greedy, selfish, and inconsiderate (albeit to differing degrees). We also have the qualities of generosity, altruism, and empathy, again to varying degrees. None of these are "good" or "bad" but are parts of the whole it is to be human. A fair and equitable system of governance must recognize the potential to provide benefits to society while being resistant to corruption. The stochastic nature of the selection rules and vacancy filling studied herein preclude prediction of outcomes so that those seeking favor must either apply influence to all candidates or to none. One potential weakness of the Stochastic Democracy model is the need to have sufficient volunteers to fulfill civic duty and provide service to their fellow citizens. If the local candidate pool of volunteers becomes too small, less than $0.06 \%$ of the population, then an organized cabal could fill conceivably the candidate pool with cronies. Should this occur, it may be self-correcting as previously-complacent citizens recognize the corruption, thereby encouraging a higher percentage of volunteers. It is therefore incumbent on the government to encourage civic responsibility and to recognize favorably not only those who serve, but also those who volunteer to be considered.

Perhaps no system of governance can be completely perfect so long as people are involved and making decisions. No one person has perfect judgment, and no one person has the good of everyone equally in mind in all that they consider. As Lord Acton quoted: "power corrupts and absolute power corrupts absolutely", so the concept of a "benign dictatorship" exists only in the imagination. As technology advances and as space travel and space settlement become more widely available there will be communities sufficiently large and diverse where the residents call for a representative form of governance. Stochastic Democracy is put forward as a suitable candidate, being not perfect, but perhaps as near unto the ideal as humans are capable of achieving. Before realizing that exciting future when large communities live outside our fragile biosphere there may arise opportunities on earth which can provide real-world testing grounds for possible further refinement and bug-fixing in the algorithms.

Researchers interested in exploring these matters are encouraged to contact the author.

\section{Acknowledgement}

The author is grateful to Beth Schubert who provided many critical comments on this work.

\section{References}

\footnotetext{
${ }^{1}$ Schubert, P.J. “A Novel System of Governance for Remote Communities,” AIAA SPACE 2010, 30 Aug - 2 Sept, 2010, Anaheim, CA.

${ }^{2}$ Schubert, P.J. “Start-up and Scale-up of a Novel Form of Colony Governance,” AIAA Space 2013, 9-11 Sept

2013. San Diego, CA.

${ }^{3}$ MATLAB, by The Mathworks, Inc., Natick, MA.
} 